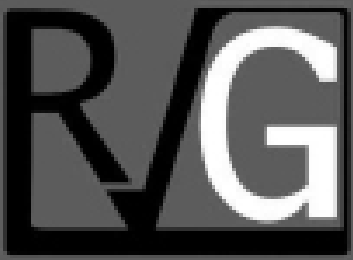

Año 24 No. 88

Octubre - Diciembre 2019

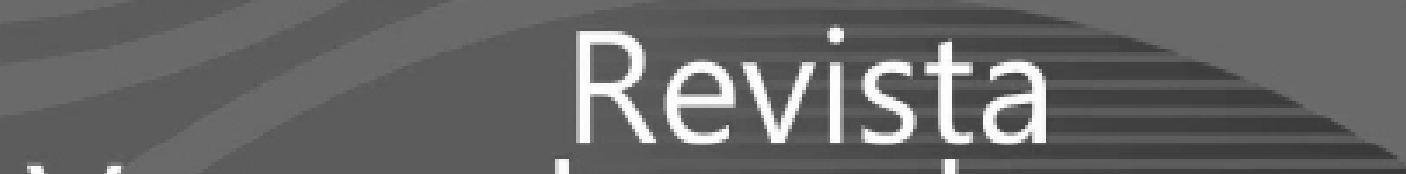

Venezolana de

verencla

UNIVERSIDAD DEL ZULIA (LUZ)

Facultad de Ciencias Económicas y Sociales

Centro de Estudios de la Empresa 


\title{
Evolución del papel del emprendedor rural: del agricultor subordinado del siglo XVIII al empresario rural actual ${ }^{1}$
}

\author{
Arias, Francisco ${ }^{2}$ \\ Ribes-Giner, Gabriela ${ }^{3}$
}

\section{Resumen}

Esta revisión muestra la evolución del emprendedor rural desde mediados del siglo XVIII hasta el presente. El objetivo principal fue analizar los cambios en los roles de los empresarios rurales debidos al contexto económico. Para ello se realizó una búsqueda bibliográfica; tomando como criterio de búsqueda el emprendimiento agrícola y rural, el período abarcó desde 1756 hasta 2018; utilizando bases de datos como Scopus, Web of Sciences, Journal Storage y Google Académico. Los hallazgos muestran su paso de agricultor subordinado a propietario, luego emprendedor y actualmente emprendedor social rural, aunque su negocio no sea necesariamente agrícola. Este emprendedor se basa actualmente en la pluriactividad y el desarrollo de redes, buscando el beneficio personal y el de la comunidad. Esto muestra que los cambios en el entorno económico promueven la adaptación del empresario rural al asumir más de un rol en la búsqueda del éxito y la sostenibilidad.

Palabras clave: empresas agrícolas; emprendimiento rural; beneficios personales; entorno económico.

Recibido:29-04-19 Aceptado: 13-09-19

1 Los autores del presente trabajo agradecen a Ph.D. Fabrice Vaillant, investigador vinculado a CIRAD (Centre de coopération internationale en recherche agronomique pour le développement); por su apoyo en la traducción y comprensión de textos del francés antiguo.

2 Programa de Doctorat en Administració i Direcció d'Empreses, Universitat Politècnica de València, Camí de Vera, s/n, 46022 València, Valencia, España. Docente Corporación Universitaria Americana. Email: fraarvar@doctor.upv.es

3 Facultat d'Administració i Direcció d'Empreses, Universitat Politècnica de València, Camí de Vera, s/n, 46022 València, Valencia, España 


\title{
Evolution of the role of the rural entrepreneur: from the subordinate farmer of the 18th century to the current rural entrepreneur
}

\begin{abstract}
This review shows the evolution of the rural entrepreneur from the mid-18th century to the present. The main objective was to analyze the changes in the roles of rural entrepreneurs due to the economic context. For this, a bibliographic search was carried out; taking agricultural and rural entrepreneurship as a search criterion, the period covered from 1756 to 2018; using databases such as Scopus, Web of Sciences, Journal Storage and Google Scholar. The findings show his step from subordinate farmer to owner, then entrepreneur and currently rural social entrepreneur, although his business is not necessarily agricultural. This entrepreneur is currently based on multi-activity and network development, seeking personal and community benefit. This shows that changes in the economic environment promote the adaptation of the rural entrepreneur by assuming more than one role in the search for success and sustainability
\end{abstract}

Keywords: agricultural companies; rural entrepreneurship; personal benefits; economic environment.

\section{Introducción}

La agricultura ha sido el motor del progreso de la humanidad; estando presente en la evolución histórica, social y económica del desarrollo humano. En una mirada al siglo dieciocho, se observa que quienes poseían la tierra, comúnmente terratenientes o señores feudales, unidos a un reino, lucharon por expandir su dominio para mejorar sus niveles de producción y acumular riqueza (Mazoyer y Roudart, 2007). El sistema feudal entra en crisis en el siglo XVI hasta el siglo XVIII; debido a las luchas de los trabajadores de la tierra, en su búsqueda de acceso a la propiedad privada y los sucesivos acontecimientos que motivaron la revolución francesa (Banaji, 1976).

Estos cambios sucesivos de sistemas en el dominio de la tierra, reconfiguraron la distribución de la población en las áreas rurales; desde un alto porcentaje de la población ubicada en la ruralidad a principios del siglo XIX (Galpin, 1918) generando una disminución de la población, debido a la migración a áreas urbanas, la cual fue motivada por la búsqueda de mejores oportunidades económicas durante el siglo XX (Casson y Casson, 2014; Farley, 1937; De Jong y Fawcett, 1981; Mazumdar, 1987; Frederick y Long, 1989; Watkins, 1907). Estos escenarios han hecho necesario que los empresarios 
agrícolas cambien continuamente sus roles en el proceso de creación de una empresa y su sostenimiento en el tiempo; especialmente en un entorno rural menos competitivo en comparación con los entornos urbanos (Harpa y Moica, 2014; Hirano, 2016; Kalantaridis y Labrianidis, 2004; Rutten, 1986; Zaremohzzabieh et al, 2016).

Debido al reducido número de estudios que analizan los roles del empresario rural y su impacto en el desarrollo rural (Pato y Teixeira, 2016); el objetivo del presente trabajo fue analizar los cambios en el papel del empresario rural, durante su adaptación al contexto económico desde el siglo XVIII y su contribución al desarrollo económico.

Para su desarrollo se propuso la siguiente metodología: la cual se orientó hacia la recolección en profundidad, basado en la propuesta de DiazMadroñero y Col (2014) y Codina
(2007); los cuales usaron matrices de referencias bibliográficas y análisis de contenidos. Las palabras "Entrepreneur", "Entrepreneurship", "Rural" and "Agriculture" fueron usadas como expresiones de búsqueda, utilizando bases de datos de reconocida calidad académica como Scopus (SC) y Web of Sciences (WS) para el periodo 1756 a 2018, también se emplearon bases de datos adicionales como Journal Storage (JS) y Scholar Google (SG) para obtener información de mayor antigüedad.

El número de referencias obtenidas fue de alrededor de 5220 resultados para el periodo (gráfico 1). Entonces para hacer manejable estos resultados, se filtraron eliminando aquellos títulos incorrectamente citados, copias múltiples del mismo documento en forma similar a lo reportado por Adriaanse y Rensleigh (2013); Falagas et al. (2008); así como Pato y Teixeira (2016).

\section{Grafico 1 \\ Publicaciones sobre emprendimiento rural en diferentes bases de datos}

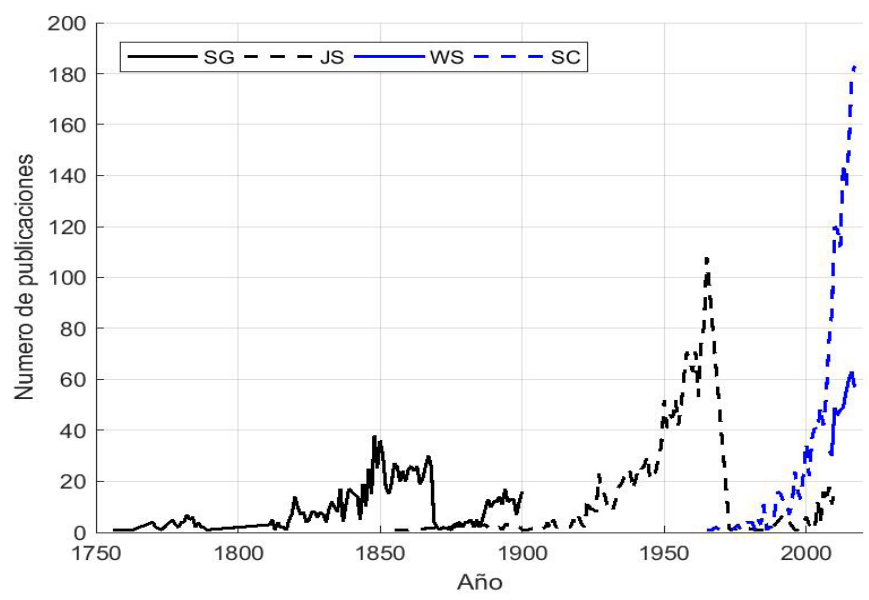

Fuente: elaboración propia 
Finalmente; se revisaron los resúmenes que hacían alusión al término de emprendimiento agrícola y lo emprendimiento rural como criterio de filtrado, obteniendo un total de 443 documentos.

\section{Evolución del rol del emprendedor a lo largo del tiempo}

De acuerdo a la revisión de la literatura se encontró que el rol del emprendedor rural, desde 1756, ha cambiado a lo largo de la línea de tiempo, adaptándose al contexto y el mercado. En este sentido, se detectaron tres roles principales (Tabla 1).

\begin{tabular}{lccc}
\multicolumn{2}{c}{ Tabla 1 } & \\
Roles del emprendedor rural reportados en la literatura \\
\hline Rol & Periodo & \multicolumn{2}{c}{ Autor } \\
\cline { 3 - 4 } & $1400-1756$ & Cantillon (1756) & Bergin (2002) \\
\hline Agricultor subordinado & $1756-1939$ & Wallerstein (1979) & Schults (1939) \\
Agricultor negociante & $1930-2019$ & Nourse (1930) & Ye y col. (2019) \\
Emprendedor rural & &
\end{tabular}

Fuente: elaboración propia

Estos diferentes roles algunas veces coexisten haciendo difícil una correcta distinción entre cada uno de ellos. El desarrollo e implicaciones de estos roles son detallados en las siguientes subsecciones.

\subsection{Agricultor subordinado}

La agricultura ha sido un pilar fundamental en el desarrollo económico de las naciones, la historia resalta que aquellos que tenían mejores niveles de producción, asociado a mejores tierras; ostentaban un mayor poderío económico, lo cual también motivo conflictos por parte de aquellos que querían nuevas tierras y riquezas para la expansión de su poder e influencia (Mazoyer y Roudart, 2007).

La aparición de terratenientes, quienes concentran la tierra en pocas manos para fines agrícolas, firmó la relación de intermediación financiera entre el estado y la base campesina, compuesta principalmente de sirvientes y personas libres (Figura 1) (García y Carlos, 2006). Los campesinos y su capacidad de producción estaban subordinados a un sistema feudal en el que no tenían acceso a la propiedad de la tierra y debían rendir tributo (Wallerstein, 1979; Boutruche, 1995). 


\section{Figura 1 \\ Estructura social en feudalismo}

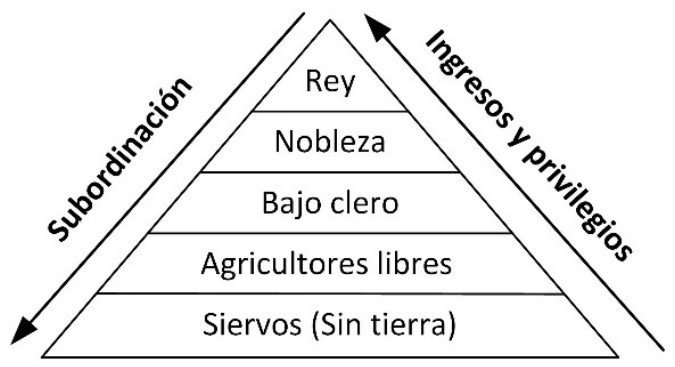

Fuente: elaboración propia basado en Monge (2001) y Stavenhagen (1969)

En este escenario; el señor feudal pertenecía a la nobleza y era quien tomaba las decisiones sobre lo que debía producirse (Cervera, 2008; Danvila, 1779), por lo cual fue considerado una especie de director de empresa agrícola (Scott, 1915), tomando su parte y haciendo la correspondiente contribución a la corona (Martín, 2018).

El desarrollo de la agricultura comercial, que buscaba producir excedentes a través de campesinos libres "cuyos impuestos, rentas y diezmos constituían la mayor parte de los ingresos del estado y la nobleza terrateniente" (Bergin, 2002), generó una tensión en contra del sistema feudal y el conflicto de clases en el mundo rural. Estos hechos conducen a un largo y lento proceso de transición del feudalismo al capitalismo, iniciado en el siglo XVI hasta el siglo XVIII (Del Rio, 2010; Stern, 1987). El descontento de la población, sumado al impulso antimonárquico contribuido en el período de la ilustración, permite que pensadores como Mirabeau desarrollen una crítica contra el papel del agricultor y su tributación hacia los propietarios y el mismo estado, mencionando que:

"El interés del propietario incluye el del estado y el uno, y el otro va en contra de sus intereses más queridos intereses, enviando o desviando los beneficios del agricultor. Esta razón afecta a todos los agricultores, ya sea un empresario o un trabajador de cultivo" (Mirabeau, 1763)

Este sentimiento permaneció latente $y$ se manifestó durante la revolución francesa cuando, entre otras cosas, se reclamó la liberación del campesino y sus tierras (Luna, 2002); lo que permitió el aumento del número de campesinos propietarios (Calatrava, 1980; Singelman y Vogan, 1981).

En este sentido, Cantillon (1756), a quien se le atribuye la primera mención del término "emprendedor", dijo: "el agricultor es un emprendedor que se compromete a pagar al propietario por la granja o la tierra una cantidad fija de dinero, sin ser seguro de la ventaja que tiene"; donde el hecho de operar en un entorno de incertidumbre convierte al individuo en un tomador de riesgos (Casson, 2010; Kanbur, 1980; Mahmood, 2009; McElwee, 2008; Stathopoulou et 
Evolución del papel del emprendedor rural: del agricultor subordinado del siglo XVIII al empresario rural actual

Arias, Francisco y Ribes-Giner, Gabriela

al, 2004).

El desarrollo de las revoluciones burguesas y los procesos de industrialización entre los siglos XVII y XIX en Europa y sus efectos en América llevaron a "desarrollar el modo de producción capitalista" (Aparicio, 2013). En este contexto, los agricultores podrían trabajar como trabajadores o inquilinos $y$, a veces, obtener suficiente dinero para comprar una parcela de tierra y convertirse en propietarios (Carver, 1911), y luego procurar excedentes que le permitieran venderlos al mercado para crecer en la medida que tomaba buenas decisiones (Galpin, 1918; Ise, 1920).

\subsection{Granjero negociante}

La industrialización de las ciudades en el siglo XIX condujo al progreso económico; en la medida en que participaron y concentraron el desarrollo técnico, científico, cultural, financiero y de servicios (Aparicio, 2013; Frankel, 1968); mientras que las áreas rurales se estancaron y aislaron en su papel de ser proveedores de productos básicos con poco o ningún valor agregado (Harris, 1969; Tauger, 2010).

Así; las empresas rurales se basaron en la producción agrícola destinada a centros urbanos y orientada hacia lo local, donde la empresa era vista como una granja y donde la estructura empresarial era difusa (Carver, 1911). En este contexto, el empresario rural era un campesino aislado, centrado en su granja, con condiciones físicas y conocimientos de manejo de cultivos para hacer prosperar su negocio (Wargner, 1887; Harris, 1969).

En el mismo período, el desarrollo de la agricultura científica y la mecanización agrícola permitió la búsqueda de mayores rendimientos
(Bernard, 1917; Harkness, 1928); donde algunos agricultores se convirtieron en empresarios que pensaban en el mercado (Foster, 1953; Galpin, 1918; Sanders, 1940; Thompson, 1921), asumiendo riesgos para obtener un posible beneficio (Petrin y Ganon, 1997; Schultz, 1939; Tuttle, 1927; Bryant, 1989; Harris, 1969).

Por lo tanto, el avance del capitalismo promovió que el agricultor debería comprar tierras y recursos, organizarlos y aprovecharlos al máximo (Tuttle, 1927). Además, invertir el capital en reposo en aquellas operaciones que generan rentabilidad (Stewart et al, 1919); buscando tomar las mejores decisiones para continuar aumentando las ganancias y controlar sus costos y recursos (Ise, 1920; Maxwell, 1929).

En este sentido, el negocio agrícola ya no se concebía como aquel en el que el agricultor esperaba que su producción fuera solicitada por el consumidor (Wargner, 1887); por el contrario, se contemplaba que el individuo se convertiría en un hombre de negocios que toma decisiones y cuya producción tendría fines comerciales (Galpin, 1918), buscando que los negocios rurales fueran similares a los que se desarrollaron en las ciudades (Bernard, 1917). Todos estos requisitos requieren de un agricultor capacitado y motivado como la base de empresas rurales exitosas (Butterfield, 1905; Sanders, 1940) y que deben ser capaces de lidiar con las fuerzas económicas y desarrollar métodos para determinar qué y cómo producir (Ise, 1920 ).

Esta capacidad de gestión es clave para la sostenibilidad del negocio rural en un entorno de incertidumbre (Mottiar et al, 2018); dónde el individuo debe ser consciente de los cambios de contexto (Foster, 1953), en consecuencia "ya no 
se trata de encontrar el sistema más productivo, sino de elegir entre una serie de estrategias que permiten un ingreso razonable" (Díaz-Pichardo y col.., 2010). Independientemente de si opera un negocio o genera una iniciativa de un nuevo tipo en un entorno rural (Leibenstein, 1968; Gladwin et al, 1989); bien sea como agricultor, emprendedor rural o empresario (McElwee, 2008).

\subsection{Emprendedor rural}

En el caso de este rol se puede dividir en las tipologías mostrada en el cuadro 1 y que se detallan en las siguientes subsecciones.

\section{Cuadro 1 \\ Tipologías del emprendedor rural}

\begin{tabular}{|c|c|}
\hline Tipo & Descripción \\
\hline I & Innovador que toma riesgos e inicia un negocio \\
\hline II & $\begin{array}{l}\text { Motivado a la pluriactividad, especialización o el desarrollo de } \\
\text { trabajos no agrícolas }\end{array}$ \\
\hline III & Constructor de redes y conexiones al mercado \\
\hline IV & Emprendedor social en la ruralidad \\
\hline V & Otras tipologías \\
\hline
\end{tabular}

Fuente: Elaboración propia

\subsubsection{Emprendedor que innova y toma riesgos}

El emprendedor, en su sentido moderno, se entiende como quién comienza un negocio y lo gestiona, combinando factores de conocimiento, capital y trabajo (Say, 2009); quién debería asumir riesgos e innovar (Schumpeter, 1934; Knight, 1921). Asi que; Durante el período 1920 - 1970, el riesgo parece impregnar la literatura sobre el espíritu empresarial cuando se establece una empresa, y no hay seguridad sobre el rendimiento de la inversión (Frederick y Long, 1989).

En estas condiciones, la nueva competitividad requiere del agricultor comprar nuevos sistemas de producción para mejorar la eficiencia de las operaciones de la granja y al mismo tiempo, realizar una adecuada gestión. Estos hechos le permiten convertirse en un riesgo crediticio deseable, posibilitando el acceso a la financiación para ampliar sus capacidades, debiendo anticipar riesgos e incertidumbres para prevalecer (Foster, 1953; Schultz, 1939; Nourse, 1930; Stigler, 1937).

En este punto, se considera que el riesgo no debe ser el factor decisivo de las ganancias del empresario (Palmäs, 2012) y debe ser el resultado de la innovación y los avances técnicos (Park, 2005); que se aclara en la siguiente definición:

"La concepción del emprendedor como agente de cambio o de la persona que utilizó factores de producción y se benefició, como resultado, se ha perdido. Hoy en día, utiliza el término emprendedor como sinónimo de una persona que comienza u opera una 
Evolución del papel del emprendedor rural: del agricultor subordinado del siglo XVIII al empresario rural actual

Arias, Francisco y Ribes-Giner, Gabriela

\section{pequeña empresa nueva. La definición de emprendimiento ha cambiado de un proceso de innovación a la fundación de un nuevo negocio" (Frederick y Long, 1989)}

Consecuentemente; es necesario definir el término "Emprendimiento rural" y a este respecto (Wortman, 1990) menciona que aún debe desarrollarse, pero se adhiere al concepto de "crear o innovar en un entorno rural". Por tanto, esto deja espacio para empresas que no son necesariamente agrícolas, pero que generan valor en las áreas rurales (Lafuente y Gómez, 2016; Pato y Teixeira, 2016). Teniendo en cuenta lo anterior, el emprendimiento rural se presenta como una secuencia de etapas que un individuo debe desarrollar, vea el diagrama 1.

\section{Diagrama 1}

\section{Etapas del emprendimiento rural}

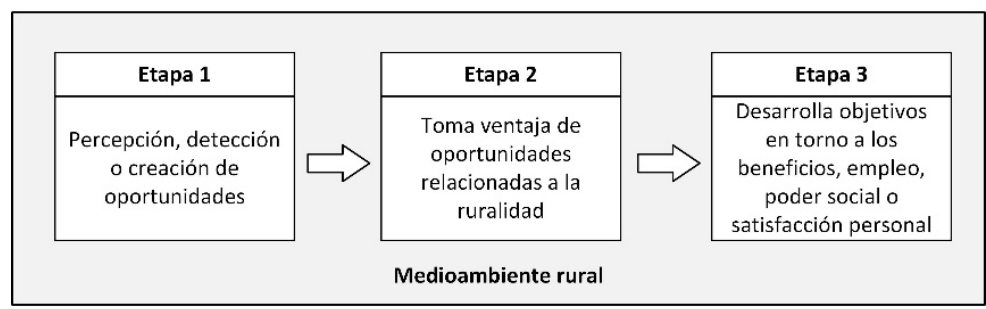

Fuente: Elaboración propia, basado en Lafuente y Gómez (2016) y Stathopoulou y Col. (2004)

A medida que la competencia avanza, se generan incertidumbres y crisis en el sistema económico actual (González, et al, 2014); lo cual requiere un mayor nivel de creatividad del empresario rural, para que su empresa pueda mantenerse vigente en el mercado (Hernández y González, 2014; Haugen y Vik, 2008). Incluso debería pensar en realizar cambios para mejorar su gestión y analizar si debe especializarse (De Lauwere, 2005) o realizar tareas adicionales para crear otros negocios complementarios que den valor a su negocio rural (Anthopoulou et al, 2017; Viladomiu y Rosell, 2016).

\subsubsection{Emprendedor motivado a la pluriactividad}

Las condiciones ambientales influyen en el individuo, en su proceso de convertirse en empresario (Harris, 1969); motivando su continua adaptación a factores externos. En este contexto, el empresario toma una idea del medio y la transforma en una oportunidad comercial (Bryant, 1989), donde las fuerzas económicas externas limitan su autonomía para la toma de decisiones (Benveuti, 1975). Por lo tanto, se requiere que el empresario sea flexible y, en ocasiones, asuma más de una función (Hisano et al, 2018; Pindado y Sánchez, 2017); en busca de nuevas fuentes de ingresos (Anderson, 2000; Georgiana et al, 2013). Estas condiciones motivan la pluriactividad, que podría definirse como "desarrollar más de una actividad, buscando diversificar o expandir" (Eikeland y Lie, 1999; Gray, 2002) (diagrama 2) 


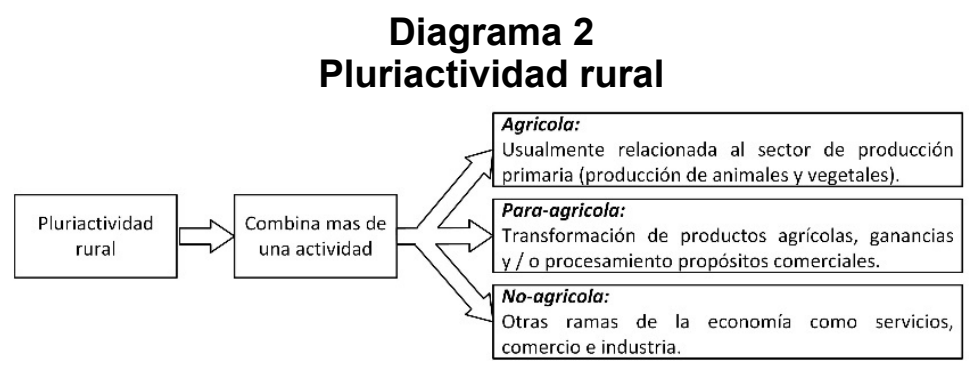

Fuente: elaboración propia, basado en Schneider (2009)

La literatura sobre pluriactividad reporta principalmente el desarrollo de actividades vinculadas al sector servicios como se comenta en el cuadro 2.

\section{Cuadro 2 \\ Actividades unidas a pluriactividad}

\begin{tabular}{ll}
\hline \multicolumn{1}{c}{ Actividad } & \multicolumn{1}{c}{ Fuente } \\
\hline Turismo rural & Fortunato (2014); Urquijo y col. (2017) \\
Renta de propiedades y prácticas deportivas & Saiko y Kanno (1990) \\
Cacería, pesca, observación de aves y aventura & Eikland y Lie (1999) \\
Alojamiento y alimentación & Bijaoui y Regev (2015) \\
Cultura, arte, participación en festivales & Hialager (2017) \\
Comercio y biodiversidad & Miller y Korsgaard (2018) \\
Agricultura orgánica & Hisano y col. (2018) \\
Generación de energía y servicios ambientales & Ferguson y Hanson (2015) \\
\hline
\end{tabular}

Fuente: elaboración propia

La pluriactividad es, a veces, la respuesta para mejorar la competitividad de las empresas agrícolas (Haugen y Vik, 2008) y no agrícolas; desarrollado en espacios rurales. Esto deja espacio para un nuevo tipo de empresario llamado "empresario rural no agrícola" (McElwee, 2008).

A lo largo del tiempo, algunos empresarios rurales han asumido diferentes roles durante la creación de una empresa y su sostenibilidad. Esto se convierte en un desafío constante, dado que la ruralidad es menos competitiva en comparación con los entornos urbanos (Harpa y Moica, 2014; Hirano, 2016; Kalantaridis y Labrianidis, 2004; Qiu et al, 2016; Rutten, 1986; Zaremohzzabieh et al, 2016).

Así; el espíritu empresarial rural se muestra como una fuerza de revitalización capaz de promover la búsqueda de 
oportunidades para empresarios locales o foráneos, motivándolos a quedarse (Gorbuntsova et al, 2018; Lan et al, 2014; Sanchis et al, 2016). Esto fomenta los procesos de contraurbanización de las personas que buscan cambiar su estilo de vida y la conexión entre lo urbano y lo rural, para generar desarrollo económico (Anthopoulou et al, 2017; Stockdale, 2016).

\subsubsection{Emprendedor que construye redes y conexiones al mercado}

La concepción de un individuo aislado en una economía de mercado no es sostenible, por lo que se requieren otros agentes que permitan la conexión y el desarrollo de sinergias para establecer una empresa rural exitosa. En última instancia, este tendrá el potencial de crecer, mantenerse y contribuir al desarrollo económico y social. Así, las redes permiten a los empresarios rurales interactuar dentro y fuera del territorio, permitiendo la transferencia de dos maneras: suministros, información, cooperación, innovación, conocimiento, habilidades, capital social, tecnología y recursos. También mejora la conectividad con el mercado y estimula la revitalización rural (Freire-Gibb y Nielsen, 2014; Munkejord, 2017; Pindado y S'anchez, 2017; Ring et al, 2010; Shu et al, 2018).

En este caso, la construcción e inserción del individuo en redes puede tener diferentes orígenes y motivaciones; en búsqueda de la competitividad de las apuestas empresariales en la ruralidad. Se pueden mencionar algunos ejemplos en Italia, donde se construyen redes basadas en vínculos fraternales (J. Schneider y Schneider, 1976); en China, promoviendo el espíritu empresarial a través del linaje y la estructura de Guanxi (Peng, 2005) y en India, basado en el sistema de castas para empresas rurales en entornos de conflicto (Bal y Judge, 2001).

Las redes permiten corregir los desequilibrios en el mercado de las personas de aquellos individuos que no tienen la capacidad, el volumen y la posibilidad periodica para proveer productos y servicios desde la ruralidad en forma constante; por lo cual el apoyo de los actores de la red es fundamental para generar la confianza con los clientes, mientras se establece la debida reputación (Poon et al, 2012). Debido a esto, grupos vulnerables, como las mujeres, han encontrado en estos mecanismos la posibilidad de superarse, los pequeños productores han podido apoyarse y complementarse entre sí, y los grupos de jóvenes encuentran la posibilidad de aprender e insertarse al mercado laboral (Korsgaard y col.., 2015), al igual que los discapacitados y otros grupos poblacionales, (Gonzáles y otros, 2014; Hustedde, 2018).

En consecuencia, las redes apoyan el establecimiento de empresas, la generación de recursos y la extracción de valor del medio rural (Anderson, 2000). Esta acción, favorecida por el aumento de la cobertura de Internet en las zonas rurales, permite la visibilidad de los territorios rurales y sus empresas (Onitsuka y Hoshino, 2018), conectando entornos rurales y periurbanos de ruralidad con centros urbanos (Batool y Ullah, 2018; Blanchard, 2017; Mayer et al, 2016; Viladomiu y Rosell, 2016).

Algunos autores mencionan el papel de las Tecnologías de la Información y la Comunicación (TIC) en la promoción del desarrollo territorial y económico (Acs, 2006; Akguèn et al, 
2011; Boza et al, 2016; Koyana y Mason, 2017; Morris y James, 2017; Ratten, 2018; Zaremohzzabieh et al, 2016), motivando la repoblación y revitalización de las áreas rurales (Akguèn et al, 2011; Anthopoulou et al, 2017; Besser y Miller, 2013). Por tanto, las personas fuera de la ruralidad ven la posibilidad de desarrollar negocios y mejorar su calidad de vida (Hansson y Kokko, 2018; Kordel, 2016; Migliore et al, 2015; Mottiar et al, 2018). Adicionalmente; se crean oportunidades para personas desplazadas, migrantes y jóvenes (Arrighetti et al, 2016; Astromskien et al, 2014; Lafuente y G'omez, 2016; Muresan et al, 2017; Satyalakshmi, 2017) o personas mayores que deseen contribuir con sus conocimientos y experiencias al entorno (Bosch y Gómez, 2010; Haga, 2017).

Finalmente, se entiende que las presiones y condiciones del mercado cambian (Arias, 2014) y el empresario rural debe tener un papel activo en la red a la que pertenece; buscando conectarse con otras redes para mejorar su capital social. Esto contribuye a su búsqueda de "construir una red de redes" (Marchante et al, 2007) que facilite la competitividad en un contexto de crisis (Aller y Grant, 2018; Luchtenberg y $\mathrm{Vu}, \mathrm{2015}$ ), reduciendo la brecha laboral y mejorando sus perspectivas (Anthopoulou et al, 2017).

\subsubsection{El emprendedor social en la ruralidad}

En gran medida, la percepción tradicional de la empresa capitalista, como aquella que prospera a expensas de la comunidad y el medio ambiente (Porter y Kramer, 2019), ayudo a fomentar el surgimiento del emprendimiento social (Kuratko et al, 2015). Esto permite generar iniciativas y empresas que crean valor social (Peredo y McLean, 2006), permitiendo la cohesión e inclusión (Stanescu et al, 2013), promoviendo la innovación (Richter, 2017), reconociendo y resolviendo problemas sociales $y$ contribuyendo al desarrollo de territorios marginados, como las zonas rurales (Núñez, 2010).

Sin embargo; algunos problemas de las empresas sociales son su tamaño y área de influencia, lo que dificulta su crecimiento (Leadbeater, 1997). Por lo tanto, el empresario debe establecer conexiones para acceder a los medios y recursos adecuados (Steiner y Teasdale, 2016), procurando que la iniciativa prospere y sea sostenible, en la medida que se integra con el mercado (Ratten, 2018); el cual generalmente presenta fallas (Santos, 2012). En este sentido (Migliore et al, 2015) considera que: "Las situaciones en las que se produce una falla del mercado son a menudo donde los emprendedores sociales juegan un papel importante debido a la incapacidad del gobierno para abordar los temas relevantes debido a la falta de conocimiento, incluso cuando cuenta con los recursos adecuados".

Dado esto, los emprendedores sociales a menudo se ven a sí mismos como héroes (Murtagh, 2017); con diferentes roles (diagrama 3 ) resolviendo los problemas de la comunidad y los entornos en los que viven o se conectan por alguna razón. Buscan identificar brechas y oportunidades desde una perspectiva altruista para impulsar el negocio, donde un beneficio personal no es su objetivo esencial, haciendo que las comunidades participen en los beneficios y acciones que motivaron a la empresa (Aileen-Boluk y Mottiar, 2014; Thompson, 2002). 


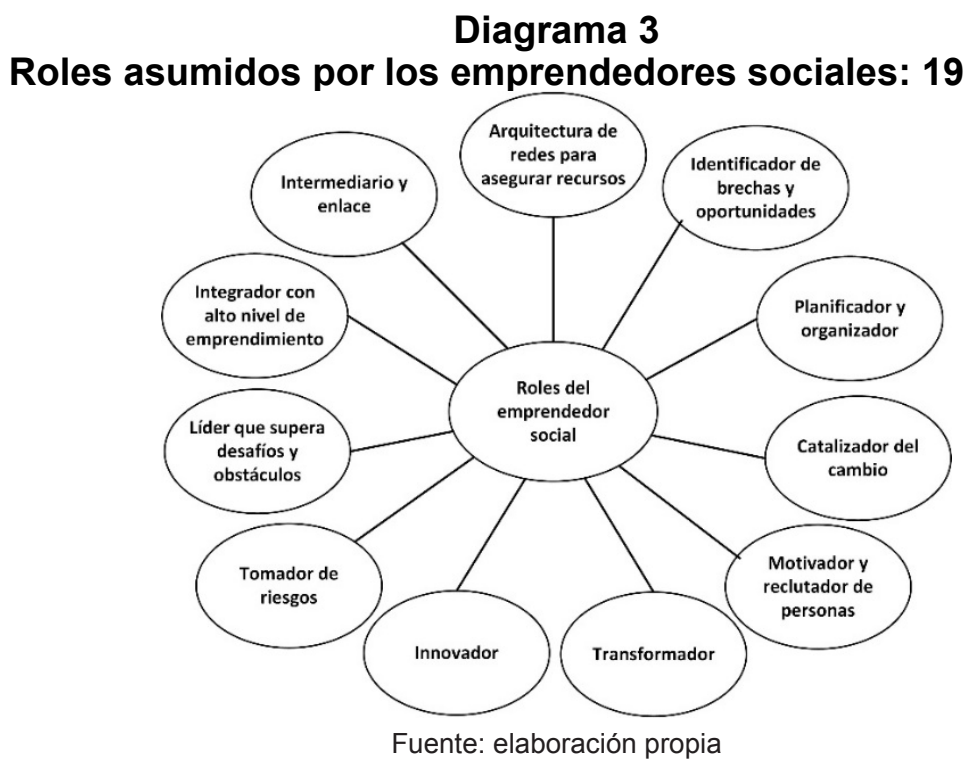

En este sentido, Porter y Kramer (2019), desarrollando su visión del emprendimiento social a nivel individual y empresarial en organizaciones establecidas, reclama el desarrollo del capitalismo con un propósito social y menciona que: "Las empresas no son los únicos actores que encuentran soluciones rentables para los problemas sociales. Toda una generación de emprendedores sociales es pionera en nuevos conceptos de productos que satisfacen las necesidades sociales utilizando modelos comerciales viables. Debido a que no están encerrados en el pensamiento empresarial tradicional, los emprendedores sociales suelen estar muy por delante de las empresas establecidas al descubrir estas oportunidades".

Igualmente; hay literatura que destaca el papel de los empresarios de edad adulta, capaces de transferir conocimientos, experiencias, recursos y capital social al desarrollo de empresas y la revitalización de la ruralidad (Bosch y Gómez, 2010; Haga, 2017; Murtagh, 2017; Walsh y O'Shea, 2008). Esto muestra a los jóvenes como una promesa para el desarrollo de este tipo de iniciativas. Los jóvenes como los que pertenecen a una "Generación Y", nacidos entre 1980 y 2000 y conocidos como "mileniales" (Calic y Mosakowski, 2016; Kuratko et al, 2017; Pasi et al, 2017; Stecker, 2014), buscan dar sentido a sus vidas compartiendo valores como el respeto por el medio ambiente y el apoyo a causas justas (Koe et al, 2012; $\mathrm{Ng}$ et al, 2010). Estas características los convierten en un terreno fértil para el emprendimiento; existiendo estudios sobre su migración a áreas rurales (Hisano et al, 2018; Sharma, 2017; Zampetakis y Kanelakis, 2010).

En consecuencia, hay varios grupos de edad que promueven el emprendimiento social en áreas rurales; 
favoreciendo la recuperación económica de las áreas en crisis (Arrighetti et al, 2016; Stanescu et al, 2013), mejorando la capacidad de resiliencia de la comunidad para enfrentar las presiones del medio (Marquis, 2009) y permitiendo entornos favorables para atraer nuevos miembros que contribuyan al proceso de revitalización rural (Avramenko y Silver, 2010; Hedlund et al, 2017; Urquijo et al, 2017).

\subsubsection{Otras tipologías de emprendedores}

Este artículo ha procurado relacionar hasta el momento, la literatura sobre el emprendimiento rural con el periodo histórico en el cual se desarrolla; sin embargo, se nota a partir del año de 1966; surgen varios autores que abordan el emprendimiento rural desde diferentes perspectivas, sin que se pueda establecer una conexión cualitativa con la coyuntura económica en la cual se desarrollan.

Al respecto hay obras de autores como Narayana (1966), Leibenstein, (1968), Lauwere y Verhaar, (2002) y McElwee, (2008); que han buscado realizar clasificaciones basadas en la tipología de los emprendedores rurales (cuadro 3).

\section{Cuadro 3}

Tipologías de emprendedores rurales

\begin{tabular}{|c|c|c|}
\hline Tipo & Descripción & Fuente \\
\hline $\begin{array}{l}\text { Innovador } \\
\text { Imitador } \\
\text { Fabianista } \\
\text { Zanganos } \\
\text { Parasitos }\end{array}$ & $\begin{array}{l}\text { Buscando oportunidades } \\
\text { Adopta innovaciones exitosas } \\
\text { Cauteloso y esceptico } \\
\text { Negativo a tomar ventaja de las oportunidades } \\
\text { Toma ventaja del gobierno para su beneficio }\end{array}$ & $\begin{array}{c}\text { Narayana } \\
(1966)\end{array}$ \\
\hline $\begin{array}{l}\text { Emprendedor rutinario } \\
\text { Nuevo tipo de emprendedor }\end{array}$ & $\begin{array}{l}\text { Realiza actividades relacionadas a la coordinación } \\
\text { y mantenimiento de una granja } \\
\text { Realiza actividades necesarias para crear o desa- } \\
\text { rrollar una compañía }\end{array}$ & $\begin{array}{l}\text { Leibenstein } \\
\text { (1968) }\end{array}$ \\
\hline $\begin{array}{l}\text { Económico } \\
\text { Socialmente responsable } \\
\text { Tradicional } \\
\text { Nuevos productores } \\
\text { Hombres de negocios dudosos }\end{array}$ & $\begin{array}{l}\text { Crea cambios económicos significantes } \\
\text { Reconoce que el éxito debe ser balanceado con } \\
\text { un rol social y medioambiental } \\
\text { Productor enfocado en una actividad que garanti- } \\
\text { za ser exitosa } \\
\text { Aquellos que diversifican en nuevos pero similares } \\
\text { áreas de su actividad } \\
\text { Renuentes al cambio }\end{array}$ & $\begin{array}{l}\text { Lauwere y Ver- } \\
\text { haar }(2002)\end{array}$ \\
\hline $\begin{array}{l}\text { Agricultor como Agricultor } \\
\text { Agricultor como emprendedor } \\
\text { Agricultor como contratista } \\
\text { Emprendedor rural, no Agricultor }\end{array}$ & $\begin{array}{l}\text { Agricultor cuya actividad económica esta basada } \\
\text { en trabajo en la tierra } \\
\text { Innovador, orientado a la oportunidad, cambios, } \\
\text { flexibles y diversas actividades económicas } \\
\text { Agricultor comprometido con labores especificas } \\
\text { Emprendedor quien ha dejado de cultivar para } \\
\text { enfocarse en negocios alternativos que no están } \\
\text { basados en la producción agrícola }\end{array}$ & $\begin{array}{l}\text { McElwee } \\
(2008)\end{array}$ \\
\hline
\end{tabular}

Fuente: elaboración propia 
El último trabajo detectado en el cual se buscó desarrollar una clasificación de las tipologías del emprendedor rural fue el de McElwee (2008), posterior a esto, no se han procurado nuevos estudios para generar un abordaje integrador, notándose que en la literatura existente entre 1990 a 2018, los investigadores centran su interés en conocer nuevos roles de los emprendedores en la ruralidad, más que de una clasificación en sí.

Se puede evidenciar que los temas más investigados son la pluriactividad, el emprendimiento social, la inserción y la construcción de redes y estilo de vida; siendo el último de mayor interés por parte de la comunidad académica (Gráfico 2)

\section{Grafico 2 \\ Roles asumidos por los emprendedores sociales}

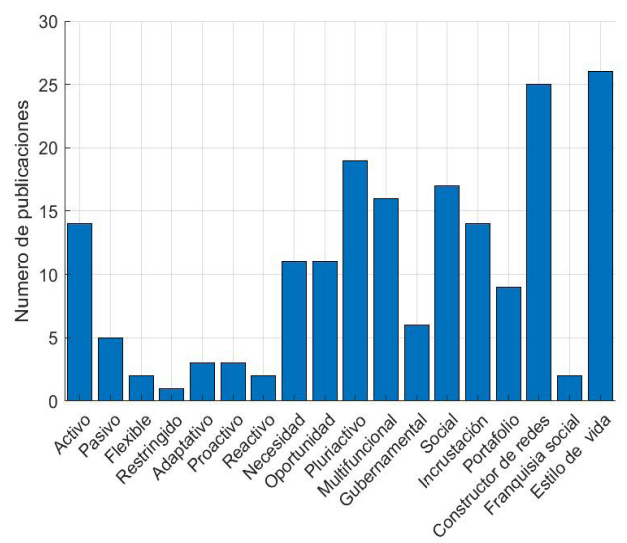

Fuente: elaboración propia basados en Leadbeater (1997); Thompson (2002); Mottiar y col. (2018); Ridha y Wahyu (2017); Lan y col. (2014)

\section{Conclusiones}

El papel del empresario rural ha evolucionado permanentemente; generando cuatro tipologías según el contexto y el mercado. Estas tipologías están incluidas en la literatura de los últimos 250 años; que van desde el campesino subordinado de la era feudal hasta el empresario rural actual. Este último puede tener una o más subtipos. Igualmente; Desde los años 90 hasta el presente, se observa el desarrollo de una diversidad de temas relacionados con las tipologías de emprendimiento rural, como los relacionados con el emprendimiento social, la inserción y la construcción de redes y el estilo de vida de los más interesantes en relación con el emprendimiento rural. También se puede mencionar que la visión del emprendimiento rural inherentemente relacionada con la agricultura ha cambiado con el desarrollo de la 
literatura; para acomodar otros tipos de actividades que no son necesariamente agrícolas, pero que tienen lugar en un espacio rural. Un ejemplo de esto son las empresas que están ubicadas en entornos rurales como servicios, industrias creativas, turismo, relacionadas con las tecnologías de la información y la comunicación, el comercio, entre otros.

\section{Referencias bibliográficas}

Acs, Zoltan (2006), How is entrepreneurship good for economic growth? Innovations:Technology, Governance, Globalization, 1 (1), 97-107. doi: 10.1162/ itgg.2006.1.1.97

Adriaanse, Leslie; Rensleigh, Chris (2013), Web of science, scopus and google scholar. The Electronic Library , 31 (6), 727-744. doi: 10.1108/ EL-12-2011-0174

Aileen-Boluk, Karla; Mottiar, Ziene (2014), Motivations of social entrepreneurs. Social Enterprise Journal, 10 (1), 53-68. doi: 10.1108/SEJ-01-20130001

Akgun, Ali; Baycan, Tuzi; Nijkamp, Peter; Poot, Jacques (2011), Roles of local and newcomer entrepreneurs in rural development: A comparative meta-analytic study. Regional Studies, 45 (9), 1207-1223. doi: $10.1080 / 00343401003792500$

Aller, Carlos; Grant, Charles (2018), The effect of the financial crisis on default by Spanish households. Journal of Financial Stability, 36 , 39-52. doi: $10.1016 /$ j.jfs.2018.02.006

Anderson, Alistair(2000), Paradox in the periphery: An entrepreneurial reconstruction? Entrepreneurship y Regional Development , 12 (2), 91-109. doi: $10.1080 / 089856200283027$

Anthopoulou, Theodosia; Kaberis, Niko- laos; Petrou, Michael(2017), Aspects and experiences of crisis in rural Greece. Narratives of rural resilience. Journal of Rural Studies, $52,1-11$. doi: 10.1016/j.jrurstud.2017.03.006

Aparicio, Abraham (2013), Historia econ'omica mundial siglos XVII - XIX: Revolu- ciones burguesas y procesos de industrializacion. Economia Informa, 378 , 60-73. doi: 10.1016/S01850849(13)71309-4

Arias, Francisco (2014), Agromarketing: una mirada al mercadeo del sector agropecuario. Caldas: Editorial Lasallista.

Arrighetti, Alessandro; Caricati, Luca; Landini, Fabio; Monacelli, Nadia (2016),En- trepreneurial intention in the time of crisis: a field study. International Journal of Entrepreneurial Behavior y Research, 22 (6), 835-859. doi: 10.1108/IJEBR-12-2015-0326

Astromskien, Adelé; Slavickien, Astrida; Gudaitien, Grazina (2014), The priorities of young rural population enterprise activity. Procedia - Social and Behavioral Sciences, 156 , 345-349. doi: 10.1016/j.sbspro.2014.11.200

Avramenko, Alex; Silver, Jane (2010), Rural entrepreneurship: Expanding the horizons. International Journal of Entrepreneurship and Innovation Management, 11 (2), 140. doi: 10.1504/ IJEIM.2010.030065

Bal, Gurpreet; Judge, Paramjit (2001), Terrorism and rural entrepreneurship in punjab. Journal of Entrepreneurship, 10 (2), 191-208. doi: 10.1177/ 097135570101000204

Banaji, Jairus (1976), The peasantry in the feudal mode of production: Towards an economic model. The Journal of Peasant Studies, 3 (3), 299-320. doi: 10.1080/03066157608437984 
Evolución del papel del emprendedor rural: del agricultor subordinado del siglo XVIII al empresario rural actual

Arias, Francisco y Ribes-Giner, Gabriela

Batool, Hira; Ullah, Kalim(2018), Pakistani women entrepreneurs and ICT intervention. Journal of Entrepreneurship Education, 21 (1), 1-15.

Benveuti, Bruno (1975), General systems theory and entrepreneureal autonomy in farming: Towards a new feudalism or towards democratic planning. Sociologia Ruralis, 15 (1-2), 46-64. doi: 10.1111/j.1467-9523.1975.tb00162.x

Bergin, Joseph (2002), El siglo XVII: Europa, 1598-1715. Grupo Planeta (GBS). Bernard, L. (1917). A theory of rural attitudes. The American Journal of Sociology , 22 (5), 630-649.

Besser, Terry; Miller, Nancy (2013), Community matters: Successful entrepreneur- ship in remote rural us locations. The International Journal of Entrepreneurship and Innovation, 14 (1), 15-27. doi: 10.5367/ijei.2013.0104

Bijaoui, Ilan; Regev, David (2015), Entrepreneurship and viral development in rural Western Negev in Israel. Journal of Research in Marketing and Entrepreneurship, 17 (1), 54-66. doi: 10.1108/JRME-09-2014-0023

Blanchard, Kevin (2017), Rural and remote SMEs' innovative behavior: Is it in the genes or location? An examination of entrepreneurial traits and characteristics. Strategic Change, 26 (4), 301309. doi: 10.1002/jsc.2132

Bosch, Pilar; Gomez, Arantza (2010), Older people as actors in the rural community, innovation and empowerment - las personas mayores como actores en la comunidad rural: Innovacion y empowerment. Athenea Digital (19).

Boutruche, Robert (1995), Señorío y feudalismo: Vínculos - dedependencia (Vol. 1). Siglo XXI.

Boza, Sofía; Marcos, Mora; Cortes, Maruja; Mora, M. (2016). Profiles based on attitudes toward public support programs of rural micro entrepreneurs of central chile. Revista de la Facultad de Ciencias Agrarias, 48 (2), 161-175.

Bryant, Christopher (1989), Entrepreneurs in the rural environment. Journal of Rural Studies, 5 (4), 337-348. doi: 10.1016/0743-0167(89)90060-0

Butterfield, Kenyon (1905), The social problems of american farmers. American Journal of Sociology, 10 (5), 606622. doi: $10.1086 / 211337$

Calatrava, Juan (1980), Estudios sobre la revolución francesa y el final del antiguo regimen (Vol. 10).Ediciones Akal.

Calic, Goran; Mosakowski, Elaine (2016),Kicking off social entrepreneurship: How a sustainability orientation influences crowdfunding success. Journal of Management Studies, 53 (5), 738-767. doi: 10.1111/joms.12201

Cantillon, Richard (1756), Essai sur la nature du commerce. Londres: $\mathrm{Chez}$ Fletcher Gyles.

Carver, Thomas (1911), Principles of rural economics. Boston: Ginn and company. Casson, M. (2010). Entrepreneurship: Theory, institutions and history. Scan-dinavian Economic History Review , 58 (2), 139-170. doi: $10.1080 / 03585522.2010 .482288$

Casson, Mark Casson, Catherine (2014), The history of entrepreneurship: Medieval origins of a modern phenomenon. Business History, 56 (8), 1223-1242. doi: 10.1080/00076791.2013.867330

Cervera, Pablo (2008), Las lecciones de econom'ıa civil, o de el comercio de bj danvila y villarrasa (1779). Cuadernos Aragoneses de Economía, 8 (1), 143-162.

Codina, Lluís; (2017), Investigación con bases de datos. Estructura y funciones de las bases de datos académicas. Análisis de componentes y estudio de 
caso. Barcelona - Spain: Universidad Pompeau Fabra.

Dávila, Bernardo (1779), Lecciones de economía civil, o de el comercio, escritas para el uso de los caballeros del real seminario de nobles.

De Jong, Gordon; Fawcett, James (1981), Motivations for migration: An assessment and a value-expectancy research model. New York: Pergamon. doi: 10.1016/ B978-0-08-0263052.50008-5

De Lauwere, Carolien (2005), The role of agricultural entrepreneurship in dutch agriculture of today. Agricultural Economics , 33 (2), 229-238. doi: 10.1111/ j.1574-0862.2005.00373.x

Del Rio, Ramón (2010), La transici'on del feudalismo al capitalismo: Una transición urbana o básicamente rural? HMiC: Historia Moderna i Contemporánia (8), 179-198.

Díaz-Madroñero, Manuel; Mula, Josefa; Peidro, David(2014), A review of discrete-time optimization models for tactical production planning. International Journal of Production Research, 52 (17), 5171-5205. doi: 10.1080/00207543.2014.899721

Díaz-Pichardo, René; Cantu-Gonz'alez, Cecilia; Lopez-Hernandez, Patricia (2010), En- trepreneurial promotion among farmers: The early impacts. World Academy of Science, Engineering and Technology, 66 , 12301243. doi: 10.1.1.294.3143

Eikeland, Sveinung; Lie, Ivar (1999), Pluriactivity in rural norway. Journal of Rural Studies, 15 (4), 405-415. doi: 10.1016/S0743-0167(99)00010-8

Falagas, Matthew; Pitsouni, Eleni; Malietzis, George; Pappas, Georgios (2008), Comparison of pubmed, scopus, web of science, and google scholar: strengths and weak- nesses. The FASEB
Journal , 22 (2), 338-342. doi: 10.1096/ fj.07-9492LSF

Farley, Miriam (1937), Japan's unsolved tenancy problem. Far Eastern Survey, 6 (14), 153-159. doi: 10.2307/3022167

Ferguson, Richard; Hansson, Helena (2015), Measuring Embeddedness and Its Effect on New Venture Creation-A Study of Farm Diversification. Managerial and Decision Economics, 36 (5), 314-325. doi: 10.1002/mde.2671

Fortunato, Michael (2014), Supporting rural entrepreneurship: a review of con- ceptual developments from research to practice. Community Development, 45 (4), 387-408. doi: 10.1080/15575330.2014.935795

Foster, George (1953), Farm organization and management (Third ed.). Prentice Hall.

Frankel, Joseph (1968), Mobilizing rural resources for industrial development. The Journal of the Human Factors and Ergonomics Society, 10 (6), 593-598. doi: $10.1177 / 001872086801000609$

Frederick, Martha; Long, Celeste (1989), Entrepreneurship theories and their use in rural development. Economic Research Service.

Freire-Gibb, Lucio; Nielsen, Kristian (2014), Entrepreneurship within urban and rural areas: Creative people and social networks. Regional Studies, 48 (1), 139-153. doi: 10.1080/00343404.2013.808322

Galpin, Charles (1918), Rural Life. New York: The Century Co.

García, Mac; Carlos, García (2006), La transici'on del esclavismo al feudalismo y la villa esclavista. Dialogues d'Histoire Ancienne, 32 (2).

Georgiana, L., Costin, C., y Cristian, P. (2013). Entrepreneurship growth as a factor for rural areas development [Pro- 
Evolución del papel del emprendedor rural: del agricultor subordinado del siglo XVIII al empresario rural actual

Arias, Francisco y Ribes-Giner, Gabriela

ceedings paper]. In Entrepreneurship vision 2020: innovation, development sustainability, and economic growth, vols 1 and 2 (pp. 1163-1167). Int Business Information management AssocIBIMA.

Gladwin, Cristian; Long, B; Babb, Emerson; Beaulieu, Lionel; Moseley, A; Mulkey, D; Zimet, D (1989), Rural entrepreneurship: One key to rural revitalization. American Journal of Agricultural Economics, 71 (5), 1305-1314. doi: $10.2307 / 1243127$

González, Carles; Perpinya, A; Tulla I Pujol, A, y Martín, N, A and Belmonte. (2014). Social farming in catalonia (spain): Social innovation and agroeco- logical dynamization as employment for exclusion - la agricultura social en catalunya: Innovación social y dinamización agroecológica para la ocupación de personas en riesgo de exclusión. Ager (17). doi: 10.4422/ager.2014.04

Gorbuntsova, Tatiana; Dobson, Stephen; Palmer, Nicola (2018), Rural entrepreneurial space and identity. The International Journal of Entrepreneurship and Innovation, 261-272. doi: 10.1177/1465750317723220

Gray, Colin (2002), Entrepreneurship, resistance to change and growth in small firms. Journal of Small Business and Enterprise Development, 9 (1), 6172. doi: 10.1108/14626000210419491

Haga, Kazue(2017), Innovation in Rural Japan: Entrepreneurs and Residents Meeting the Challenges of Aging and Shrinking Agricultural Communities. Journal of Innovation Economics y Management

Hansson, Helena; Kokko, Suvi (2018), Farmers' mental models of change and implications for farm renewal A case of restoration of a wetland in Sweden. Journal of Rural Studies , 60 , 141-151. doi: 10.1016/j.jurstud.2018.04.006
D. A. E. Harkness (1928), Agriculture and the price level. The Economic Journal, 38 (149), 56. doi: 10.2307/2224396

Harpa, Elena; Moica, Sorina (2014), The role of innovation in shaping the identity of the entrepreneur in a rural context [Proceedings Paper]. In I. Boldea Lulian (2014), Globalization and intercultural dialogue: Multidisciplinary perspectives

- economy and management (pp. 832846). Arhipelag XXI Press.

Harris, Marshall (1969). Shifts in entrepreneurial functions in agriculture. American Journal of Agricultural Economics, 51 (3), 517. doi: 10.2307/1237906

Haugen, Marit; Vik, Jostein (2008), Farmers as entrepreneurs: The case of farm-based tourism. International Journal of Entrepreneurship and Small Business, 6 (3), 321-336.

Hedlund, Martin; Carson, Doris; Eimermann, Marco; Lundmark, Linda (2017), Repopulating and revitalising rural Sweden? Re-examining immigration as a solution to rural decline. The Geographical Journal, 183 (4), 400-413. doi: 10.1111/geoj.12227

Hernández, Rosa; González, Oscar (2014), Rural Lodging Establishments as Drivers of Rural Development. Journal of Travel Research, 53 (1), 8395. doi: $10.1177 / 0047287513481273$

Hirano, Makoto (2016), Role of an entrepreneur for innovating regional agriculture: through a case of a japanese ice cream firm. International Journal of Entrepreneurship and Small Business , 29 (2), 322-337. doi: 10.1504/ IJESB.2016.078717

Hisano, Shuji; Akitsu, Motoki; McGreevy, Steven (2018), Revitalising rurality under the neoliberal transformation of agriculture: Experiences of re-agrarianisation in Japan. Journal 
of Rural Studies . doi: 10.1016/j.jrurstud.2018.01.013

Hjalager, Anne-Mette (2017), Ruralurban business partnershipsTowards a new trans-territorial logic. Local Economy, 32 (1), 34-54. doi: 10.1177/ 0269094216686528

Hustedde, Ronald (2018), Entrepreneurship and Economic Development in Rural America. In Disability and vocational rehabilitation in rural settings (pp. 3-16). Springer International Publishing. doi: 10.1007/978-3319-64786-9 1

Ise, J. (1920). What is rural economics. The Quarterly Journal of Economics, 34 (2), 300. doi: 10.2307/1882365

Kalantaridis, Christos; Labrianidis, Lois (2004), Rural entrepreneurs in russia and the ukraine: Origins, motivations, and institutional change. Journal of Economic Issues, 38 (3), 659-681. doi: 10.1080/00213624.2004.11506724

Kanbur, Ravi; (1980), A note on risk taking, entrepreneurship, and schumpeter. History of Political Economy, 12 (4), 489-498. doi: 10.1215/00182702$12-4-489$

Knight, Frank (1921), Risk, Uncertainty and profit. Boston: Riverside Press.

Koe, Wei-Loon; Rizal, Juan; Majid, Izaidin; Ismail, Kamariah. (2012), Determinants of entrepreneurial intention among millennial generation. Procedia - Social and Behavioral Sciences, 40 , 197 - 208. doi: https://doi. org/10.1016/j.sbspro.2012.03.181

Kordel, Stefan (2016), Selling ruralities: how tourist entrepreneurs commodify traditional and alternative ways of conceiving the countryside. Rural Society, 25 (3), 204-221. doi: 10.1080/10371656.2016.1255475

Korsgaard,Steffen; Muller, Sabine; Tanvi-
g,Hanne (2015), Rural entrepreneurship or entrepreneurship in the rural between place and space. International Journal of Entrepreneurial Behavior y Research, 21 (1), 5-26. doi: 10.1108/ IJEBR-11-2013-0205

Koyana, Siphokazi; Mason, Roger. (2017), Rural entrepreneurship and transformation: the role of learnerships. International Journal of Entrepreneurial Behavior y Research, 23 (5), 734-751. doi: 10.1108/IJEBR-07-2016-0207

Kuratko, Donald; McMullen, Jeffery; Hornsby, Jeff; Jackson, Chad (2017), Is your organization conducive to the continuous creation of social value? Toward a social corporate entrepreneurship scale. Business Horizons, 60 (3), 271-283. doi: 10.1016/j.bushor.2016.12.003

Kuratko, Donald; Morris, Michael; Schindehutte, Minet (2015), Understanding the dynamics of entrepreneurship through framework approaches. Small Business Economics, 45 (1), 1-13. doi: 10.1007/s11187-015-96273

Lafuente, Esteban; G'omez, Eduardo (2016), The Territorial Economic Impact of En- trepreneurial Youthfulness. Strategic Change, 25 (2), 187-204. doi: 10.1002/ jsc.2055

Lan, Hong; Zhu, Ying; Ness, David; Xing, Ke; Schneider, Kris (2014), The role and characteristics of social entrepreneurs in contemporary rural cooperative de- velopment in China: case studies of rural social entrepreneurship. Asia Pacific Business Review, 20 (3), 379-400. doi: 10.1080/13602381.2014.929300

Lauwere, Caroline; Verhaar, Kees (2002), Het mysterie van het ondernemerschap. Wageningen: Wageningen UR.

Leadbeater, Charles. (1997), The Rise 
Evolución del papel del emprendedor rural: del agricultor subordinado del siglo XVIII al empresario rural actual

Arias, Francisco y Ribes-Giner, Gabriela

of the Social Entrepreneur. Demos. Leibenstein, Harvey(1968), Entrepreneurship and development. The AmericanEconomic Review, 58 , 72-83. doi: $10.2307 / 1831799$

Luchtenberg, Kimberly; Quang Viet(2015), The 2008 financial crisis: Stock market con- tagion and its determinants. Research in International Business and Finance, 33 , 178-203. doi: 10.1016/j. ribaf.2014.09.007

Luna, Pablo (2002), El mayor acontecimiento de la revolución: la venta de los biens nationaux en francia, a finales del siglo XVIII. Mundo Agrario, 3 (5).

Mahmood, Tariq (2009), Developing entrepreneurship for rural youth and women. In Work, learning and sustainable development. Springer.

Marchante, Joaquín Varela, Carmen; Guerrero, Ana; Navarro, José. (2007), The rural entrepreneurship within the context of territorial development. a case study. Boletin de la Asociacion de Geografos Espanoles(43).

Marquis, Greg (2009), Journal of Enterprising Communities: People and Places in the Global Economy For Authors. Journal of Enterprising Communities: People and Places in the Global Economy Iss, 3 (4), 355-368. doi: 10.1108/ 17506200910999101

Martín, Francisco (2018); Feudalismo y señor'ıo en europa. Ediciones Paraninfo, SA. Maxwell, J. (1929). An examination of some marshallian concepts (Vol. 19). American Economic Association.

Mayer, Haike; Habersetzer, Antoine; Meili, Rahel (2016), RuralUrban Linkages and Sustainable Regional Development: The Role of Entrepreneurs in Linking Peripheries and Centers. Sustainability, 8 (8), 745. doi: 10.3390/ su8080745
Mazoyer, Marcel; Roudart, Laurence (2007), A history of world agriculture: from the neolithic age to the current crisis. Routledge.

Mazumdar, Dipak (1987), Rural-urban migration in developing countries. In Handbook of regional and urban economics (pp. 1097-1128). doi: 10.1016/ S1574-0080(87)80014-7

McElwee, Gerard (2008), A taxonomy of entrepreneurial farmers. International Journal of Entrepreneurship and Small Business, 6 (3), 465. doi: 10.1504/ IJESB.2008.019139

Migliore, Giuseppina; Schifani, Giorgio; Romeo, Pietro; Hashem, Shadi; Cembalo, Luigi; (2015), Are Farmers in Alternative Food Networks Social Entrepreneurs? Evidence from a Behavioral Approach. Journal of Agricultural and Environmental Ethics, 28 (5), 885-902. doi: 10.1007/s10806015-9562-y

Mirabeau, Victor (1763). Philosophie rurale, ou economie g'en'erale et politique de l'agriculture. A Amsterdam Chez Les Libraries Associes.

Monge, Julio (2001), De señores y campesinos a poderes feudales y comunidades: Elementos para definir la articulación entre territorio y clases sociales en la alta edad media castellana. In Comunidades locales y poderes feudales en la edad media (pp. 115-156).

Morris, Wyn; y James, Penri (2017); Social media, an entrepreneurial opportunity for agriculture-based enterprises. Journal of Small Business and Enterprise Development, 24 (4), 1028-1045. doi: 10.1108/ JSBED-01-2017-0018

Mottiar, Ziene; Boluk, Karla; y Kline, Carol (2018), The roles of social entrepreneurs in rural destination development. Annals of Tourism Re- 
search, 68 , 77-88. doi: 10.1016/j. annals.2017.12.001

Sabine Müller; yKorsgaard, Steffen(2018), Resources and bridging: the role of spatial context in rural entrepreneurship. Entrepreneurship and Regional Development, 30 (1-2), 224-255. doi: 10.1080/08985626.2017.1402092

Munkejord, Mai (2017), Local and transnational networking among female immigrant entrepreneurs in peripheral rural contexts: Perspectives on russians in finnmark, norway. European Urban and Regional Studies, 24 (1), 7-20. doi: 10.1177/0969776415587122

Muresan, Iulia; Chiciudean, Gabriela; Arion, Felix; Porutiu, Andra; y Harun, Razhen (2017), Students' Attitudes and Intentions towards Rural Tourism Entrepreneurship. Balkan Region Conference on Engineering and Business Education, 3 (1). doi: 10.1515/cplbu-2017-0012

Murtagh, Brenda (2017), Ageing and the social economy. Social Enterprise Journal,13 (3), 216-233. doi: 10.1108/SEJ-02-2017-0009

Narayana, D (1966), Entrepreneurship and agricultural development. Bombay: Asian Stud Press.

Ng, Eddy; Schweitzer, Linda; y Lyons, Sean (2010), New generation, great expecta- tions: A field study of the millennial generation. Journal of Business and Psychology, 25 (2), 281292. doi: 10.1007/s10869-010-9159-4

Nourse, Edwin (1930), Some economic and social accompaniments of the mechanization of agriculture (Vol. 20). American Economic Association.

Núñez, Guadalupe (2010), Emprendimiento social: integrando a los excluidos en el ambito rural social entrepreneurship: Integrating the excluded in rural areas. Revista de Ciencias So- ciales, 16 (4), 579-590.

Onitsuka, Kenichiro; y Hoshino, Satoshi (2018), Inter-community networks of rural leaders and key people: Case study on a rural revitalization program in Kyoto Prefec- ture, Japan. Journal of Rural Studies . doi: 10.1016/j.jrurstud.2018.04.008

Palm as, K. (2012). Re-assessing schumpeterian assumptions regarding entrepreneurship and the social. Social Enterprise Journal, 8 (2), 141-155. doi: 10.1108/17508611211252855

Park, John (2005), Opportunity recognition and product innovation in en- trepreneurial hi-tech start-ups: A new perspective and supporting case study. Technovation, 25 (7), 739-752. doi: 10.1016/j.technovation.2004.01.006

Pasi, Pyöriä; Satu, Ojala; Tiina, Saari; y Katri-Maria Järvinen (2017), The millennial generation: $A$ new breed of labour? SAGE Open, 7 (1), 2158244017697158. doi: 10.1177/ 2158244017697158

Pato, María; Teixeira, Aurora (2016), Twenty years of rural entrepreneurship: A bibliometric survey. Sociologia Ruralis, 56 (1), 3-28. doi: 10.1111/soru.12058

Peng,Yusheng (2005), Lineage networks, rural entrepreneurs, and max weber. In

Steve Vallas (1999), Research in the sociology of work (Vol. 15, pp. 327-355). doi: 10.1016/S0277-2833(05)15013-4

Peredo, Ana; McLean, Murdith (2006), Social entrepreneurship: A critical review of the concept. Journal of World Business, 41 (1), 56-65. doi: 10.1016/ j.jwb.2005.10.007

Petrin, T; y Ganon, A (1997), Rural development through entrepreneurship. FAO.

Pindado, Emilio; Sánchez, Mercedes 
Evolución del papel del emprendedor rural: del agricultor subordinado del siglo XVIII al empresario rural actual

Arias, Francisco y Ribes-Giner, Gabriela

(2017), Researching the entrepreneurial behaviour of new and existing ventures in European agriculture. Small Business Economics, 49 (2), 421444. doi: 10.1007/s11187-017-9837-y

Poon, Jessie; Thai, Diep y Naybour, Deborah (2012), Social capital and female en- trepreneurship in rural regions: Evidence from vietnam. Applied Geography, 35 (1-2), 308-315. doi: 10.1016/j. apgeog.2012.08.002

Porter, Michael; y Kramer, Mark (2019), Creating shared value. In Managing sustainable business (pp. 327-350). Springer.

Qiu, Shubing; Zhou, Xiaohong; Gong, Bengang (2016), Building new farmer-entrepreneur training systems in poor relocation-settlements. International Journal of Innovation Science, 8 (1), 89-96. doi: 10.1108/IJIS-03-2016-005

Ratten, Vanessa (2018), Social entrepreneurship through digital communication infarming. World Journal of Entrepreneurship, Management and Sustainable Development , 14 (1), 99-110. doi: 10.1108/WJEMSD-07-2017-0045

Richter, Ralph (2017, dec), Rural social enterprises as embedded intermediaries: The innovative power of connecting rural communities with supra-regional networks. Journal of Rural Studies , 0-1. doi: 10.1016/j.jrurstud.2017.12.005

Ridha, Rizki; Wahyu, Budi (2017), Entrepreneurship intention in agricultural sector of young generation in Indonesia. Asia Pacific Journal of Innovation and Entrepreneurship, 11 (1), 76-89. doi: 10.1108/APJIE-04-2017-022

Ring, kirk; Peredo, Ana; Chrisman, James (2010), Business Networks and Economic Development in Rural Communities in the United States. Entrepreneurship Theory and Practice, 34 (1), 171-195. doi: 10.1111/j.1540-6520.2009.00307.x
Rutten, Mario (1986), Social profile of agricultural entrepreneurs. economic behaviour and life-style of middlelarge farmers in central gujarat. Economic y Political Weekly, 21 (13), A15-A23.

Saito, katsuyuki; Kanno, Masahide. (1990), Development of private sports facilities as a side business of urban farmers. Geographical review of Japan, Series B., 63 (1), 48-59. doi: 10.4157/ grj1984b.63.48

Sanchis, Raúl; Serrano, Vicente; y Koster, Pau (2016); Culture as a factor of socio- economic innovation in rural areas: The case of the artistic craftwork cluster of La Citt'a Europea dei Mestieri d'Arte (CITEMA) - . Ager , 2016 (20). doi: 10.4422/ager.2015.15

Sanders, Irwin (1940), Bulgarians and Southern Rural Whites in Contrast. Social Forces, 19 (1), 88-94. doi: $10.2307 / 2570848$

Santos, Felipe (2012), A Positive Theory of Social Entrepreneurship. Journal of Business Ethics, 111 (3), 335-351. doi: 10.1007/s10551-012-1413-4

Satyalakshmi, Kompella (2017), Understanding the Entrepreneurial Intentions of the Indian Millennials: A Pilot Study. Journal of Entrepreneurship Development, 14 (2).

Say, Jean (2009), A treatise on political economy with a new introduction by munir y salim rashid. NewJersey: Transaction Publisher.

Schneider, Jane; Schneider, Peter (1976), Peasants, herdsmen, and rural entrepreneurs. In Culture and political economy in western sicily (pp. 57-79). Elsevier. doi: 10.1016/B978-0-12627850-7.50010-0

Schneider, Sergio (2009), La pluriactividad en el medio rural brasileñ: caracter'ısticas y perspectivas para la investigaci'on.La pluriactividad en el campo 
pp. $1005-1028$

Revista Venezolana de Gerencia, Año 24 No. 88, 2019

latinoamericano, 207-242.

Schultz, Mary (1939), Theory of the firm and farm management research. Journal of Farm Economics, 21 (3), 570. doi: $10.2307 / 1232142$

Schumpeter, Joseph (1934), The theory of economic development. Cambridge: Har- vard University Press.

Scott, William (1915), John kells ingram, a history of political economy. Washington: Liberty Fund.

Sharma, Narayan (2017), Role and Importance of Rural Entrepreneurs in India. Journal of HR, Organizational Behaviour y Entrepreneurship Development, 1, 25-29.

Shu, Rui; Ren, Shenggang; Zheng, Yi (2018), Building networks into discovery: The link between entrepreneur network capability and entrepreneurial opportunity discovery. Journal of Business Research, 85 , 197-208. doi: 10.1016/j.jbusres.2017.12.048

Singelman, Peter; Vogan, María (1981), La transición clásica del feudalismo al capitalismo y la transformación agraria restringida bajo el capitalismo perif'erico. Revista Mexicana de Sociologia, 343-373.

Stanescu, I., Anton, A., y Cojocaru, D. (2013). Entrepreneurship in rural areas. In Fourth international conference psycho-social perspectives in the quasi-coercive treatment of offenders: social control and vulnerable groups (specto 2013) (pp. 533-537). Medimind SRL.

Stathopoulou, Sofia; Psaltopoulos, Demetrios; Skuras, Demitris. (2004), Rural entrepreneurship in europe. International Journal of Entrepreneurial Behavior y Research, 10 (6), 404-425. doi: 10.1108/13552550410564725

Stavenhagen, R. (1969). Las clases sociales en las sociedades agrarias. Siglo
XXI.

Stecker, Michelle (2014), Revolutionizing the nonprofit sector through social entrepreneurship. Journal of Economic Issues , 48 (2), 349-358. doi: 10.2753/ JEI0021-3624480208

Steiner, Artur.; Teasdale, Simon (2016), The playground of the rich? Growing social business in the 21st century. Social Enterprise Journal , 12 (2), 201-216. doi: 10.1108/SEJ-12-2015-0036

Stern, Steve (1987), Feudalismo, capitalismo y el sistema mundial en la perspectiva de am'erica latina y el caribe. Revista Mexicana de Sociolog' Ia, 3-58.

Stewart, Charles; Spilmman, W., y Hibbard, B. (1919). American economic association land tenure and public policy discussion. The American Economic Review , 9 (1), 226-232.

Stigler, George (1937), A generalization of the theory of imperfect competition. Journal of Farm Economics, 19 (3), 707. doi: $10.2307 / 1231448$

Stockdale, Aileen (2016), Contemporary and Messy' Rural In-migration Processes: Comparing Counterurban and Lateral Rural Migration. Population, Space and Place, 22 (6), 599-616. doi: 10.1002/psp.1947

Tauger, Mark (2010), Agriculture in World History. New York: Routledge. doi: $10.4324 / 9780203847480$

Thompson, John (1921), Mobility of the factors of production as affecting variation in their proportional relation to each other in farm organization. Journal of Political Economy, 29 (2), 108-137.

Thompson, John (2002), The world of the social entrepreneur. International Journal of Public Sector Management, 15 (5), 412-431. doi: 10.1108/ 09513550210435746

Tuttle, Charles (1927), The entrepreneur 
Evolución del papel del emprendedor rural: del agricultor subordinado del siglo XVIII al empresario rural actual

Arias, Francisco y Ribes-Giner, Gabriela

function in economic literature. Journal of Political Economy, 35 (4), 501-521. doi: $10.1086 / 253873$

Urquijo, Pedro; Bocco, Gerardo; Boni, Andrew (2017), New rurality and the experience of place: the small rural locality of La Niña, Buenos Aires, Argentina. GeoJour- nal . doi: 10.1007/s10708017-9834-3

Viladomiu, Lourdes; Rosell, Jordi (2016), Nova ruralitat a Catalunya a les u'ltimes d'ecades: diversificaci'o, emprenedoria i ocupaci'o.Documents d'Análisi Geográfica, 62 (3), 553. doi: 10.5565/ rev/dag.375

Wallerstein, Immanuel (1979), El moderno sistema mundial. la agricultura capitalista y los or'igenes de la econmía-mundo europea en el siglo XVII. Revista de estudios políticos (12), 223-225.

Walsh, Kieran; O'Shea, Eamon (2008), Responding to rural social care needs: Older people empowering themselves, others and their community. Health $y$ Place, 14 (4), 795-805. doi: 10.1016/j. healthplace.2007.12.006

Wargner, Amos (1887), Three phases of cooperation in the west. American Economic Association, 2 (1), 9-119.

Watkins, George (1907), The growth of large fortunes. Publications of the American Economic Association, 8 (4), 1-170.

Wortman, Max (1990), Rural entrepreneurship research: An integration into the entrepreneurship field. Agribusiness , 6 (4), 329-344. doi: 10.1002/1520

Xiao, H., y Zhou Lingqiang (2019), Small accommodation business growth in rural areas: Effects on guest experience and financial performance. Internatio- nal Journal of Hospitality Management , 76 (March 2018), 29-38. doi: 10.1016/j.jijm.2018.03.016

Zampetakis, Leonidas; Kanelakis, George (2010), Opportunity entrepreneurship in the rural sector: Evidence from greece. Journal of Research in Marketing and Entrepreneurship, 12 (2), 122142. doi: $10.1108 / 14715201011090594$

Zaremohzzabieh, Zeinad; Samah, Bahaman; Muhammad, Mahazan; Omar, Siti; Bolong, Jusang; Shaffril, Hayrol (2016), An investigation into factors influencing rural youth entrepreneurs' intentions to use ict: A case of malaysia. International Journal of Entrepreneurship and Small Business, 27 (4), 480-504. doi: 10.1504/ IJESB.2016.0750106297(199007)6:4(329::AID AGR2720060405)3.0.CO;2-N

Ye, S., Xiao, H., y Zhou, L. (2019). Small accommodation business growth in rural areas: Effects on guest experience and financial performance. International Journal of Hospitality Management , 76 (March 2018), 29-38. doi: 10.1016/j.jijhm.2018.03.016

Zampetakis, L., y Kanelakis, G. (2010). Opportunity entrepreneurship in the rural sector: Evidence from greece. Journal of Research in Marketing and Entrepreneurship, 12 (2), 122-142. doi: $10.1108 / 14715201011090594$

Zaremohzzabieh, Z., Samah, B., Muhammad, M., Omar, S., Bolong, J., y Shaffril, H. (2016). An investigation into factors influencing rural youth entrepreneurs' intentions to use ict: A case of malaysia. International Journal of Entrepreneurship and Small Business, 27 (4), 480-504. doi: 10.1504/ IJESB.2016.075010

- Esta obra está bajo una licencia de Creative Commons Reconocimiento-NoComercial- Compartirlgual 3.0 Unported. http://creativecommons.org/licenses/by-nc-sa/3.0/deed.es_ES 